\title{
Acute effects of alcohol on social and personal decision making
}

\author{
Hanna Karlsson ${ }^{1,4}$, Emil Persson (DD ${ }^{2,4}$, Irene Perini ${ }^{1}{ }^{1}$, Adam Yngve (D) ${ }^{1}$, Markus Heilig ${ }^{1,4}$ and Gustav Tinghög (D ${ }^{2,3,4}$ \\ (c) The Author(s), under exclusive licence to American College of Neuropsychopharmacology 2021
}

\begin{abstract}
Social drinking is common, but it is unclear how moderate levels of alcohol influence decision making. Most prior studies have focused on adverse long-term effects on cognitive and executive function in people with alcohol use disorders (AUD). Some studies have investigated the acute effects of alcohol on decision making in healthy people, but have predominantly used small samples and focused on a narrow selection of tasks related to personal decision making, e.g., delay or probability discounting. Here, we conducted a large $(n=264)$, preregistered randomized placebo-controlled study (RCT) using a parallel group design, to systematically assess the acute effects of alcohol on measures of decision making in both personal and social domains. We found a robust effect of a $0.6 \mathrm{~g} / \mathrm{kg}$ dose of alcohol on both moral judgment and altruistic behavior, but no effects on several measures of risk taking or waiting impulsivity. These findings suggest that alcohol at low to moderate doses selectively moderates decision making in the social domain, and promotes utilitarian decisions over those dictated by rule-based ethical principles (deontological). This is consistent with existing theory that emphasizes the dual roles of shortsighted information processing and salient social cues in shaping decisions made under the influence of alcohol. A better understanding of these effects is important to understand altered social functioning during alcohol intoxication.
\end{abstract}

Neuropsychopharmacology (2022) 47:824-831; https://doi.org/10.1038/s41386-021-01218-9

\section{INTRODUCTION}

There is a lack of systematic research on the effects of moderate alcohol intake on decision making in non-clinical populations. This may be related to the difficulties that go into designing these types of studies, and the fact that prior research has been primarily focused on the adverse consequences of alcohol use disorders (AUD) on physiology and behavior. Numerous studies have investigated impairments in interpersonal behavior and decision-making processes in patients with AUD, but these studies cannot disaggregate the direct effects of alcohol from functional consequences of alcohol-induced organ damage, such as e.g., well documented alcohol-induced regional gray matter loss in AUD [1].

In healthy volunteers, alcohol intake can influence incentive motivation through activation of canonical dopaminergic brain reward system, but these effects vary by gender and genetics [2-5]. Enhanced emotional reactivity and increased positive mood have also been linked to alcohol intake in non-threatening environments $[6,7]$. It is furthermore widely held that alcohol results in broad and non-selective impairments of cognitive function, but this notion has recently been questioned. A meta-analysis of studies that examined the effects of alcohol on event-related potentials suggests that alcohol intake results in relatively selective impairments of attention, automatic auditory processing, and performance monitoring [8]. Similarly, alcohol is commonly held to increase impulsivity, but available studies make it difficult to disentangle to what extent impulsivity is a cause vs. a consequence of alcohol use, and also point to the moderating influence of emotional states [9].
Few studies have examined acute effects of alcohol on motivated behavior and decision making under a level of experimental control that allows causal inferences. For instance, many of the existing studies have used survey data to compare the behavior of people who abuse alcohol to those who do not. Although there are also placebo-controlled laboratory studies, most of these have used small samples and focused on a narrow selection of tasks related to personal decision making, primarily risk taking and impulsivity [10-20]. Even for these tasks, there is a lack of converging evidence. Some studies found increased risk taking due to alcohol [11, 13], while others found no effect $[10,12,14,15,19,20]$. Similarly, waiting impulsivity has been found to increase [19] or decrease [16] following alcohol intake, but the majority of studies have found mixed or no effects $[10,11,14,15,17]$. Prototypical tasks for altruism and moral judgment have only been included in a minority of studies, with mixed results for both types of tasks [19-22]. In addition, some studies have used an observational field paradigm, typically approaching people in a bar with a structured questionnaire [22-24]. Whereas important insights can be obtained from these observational studies, they cannot provide answers about the causal relationship between alcohol intake and behavior, as they are inherently correlational, and also prone to selection bias.

Here, we therefore investigated how moderate acute alcohol intoxication influences basic social and personal decision making central to a wide variety of everyday behaviors: altruistic behavior and distributional preference, moral judgment, waiting impulsivity, and choice under risk. To this end, we conducted a preregistered

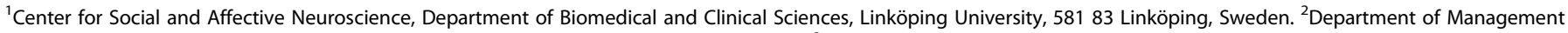

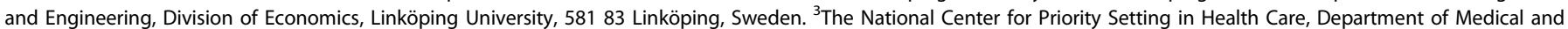

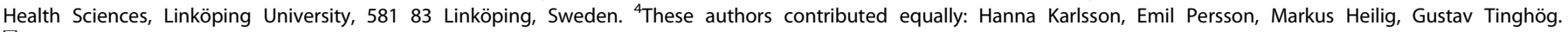

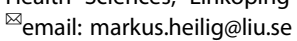


Table 1. Sample characteristics.

\begin{tabular}{|c|c|c|}
\hline & $\begin{array}{l}\text { Placebo } \\
n=128\end{array}$ & $\begin{array}{l}\text { Alcohol } \\
n=136\end{array}$ \\
\hline Female & $40 \%$ & $42 \%$ \\
\hline Age, Mean $\pm S D$, range & $\begin{array}{l}25.3 \pm 6.8 \\
20-54\end{array}$ & $\begin{array}{l}26.1 \pm 8.4 \\
20-59\end{array}$ \\
\hline Body weight, Mean $\pm S D$, range & $\begin{array}{l}74.7 \pm 13 \\
48-125\end{array}$ & $\begin{array}{l}75.6 \pm 16 \\
43-130\end{array}$ \\
\hline \multicolumn{3}{|l|}{ Education, highest level completed } \\
\hline High school & $59 \%$ & $61 \%$ \\
\hline University, $<3$ years & $17 \%$ & $12 \%$ \\
\hline University, $\geq 3$ years & $24 \%$ & $27 \%$ \\
\hline AUDIT, Mean \pm SD & $6.2 \pm 3.0$ & $6.0 \pm 3.0$ \\
\hline \multicolumn{3}{|l|}{ NEO-FFI, Mean \pm SD, $n$} \\
\hline Extraversion & $29.9 \pm 7.6,125$ & $29.0 \pm 7.0,135$ \\
\hline Neuroticism & $17.8 \pm 7.9,125$ & $18.5 \pm 8.0,134$ \\
\hline Openness & $29.0 \pm 6.5,123$ & $29.9 \pm 6.0,133$ \\
\hline Agreeableness & $34.6 \pm 6.1,123$ & $35.1 \pm 5.9,133$ \\
\hline Conscientiousness & $32.2 \pm 7.1,125$ & $31.1 \pm 7.3,130$ \\
\hline
\end{tabular}

Notes: Baseline measures for participants who completed the study. AUDIT Alcohol Use Disorder Identification Test, scores of $>20$ indicate high likelihood of dependence, NEO-FFI abbreviated Five Factor Inventory personality assessment.

(see https://osf.io/sf5em) randomized placebo-controlled study, using a general task paradigm and a substantially larger sample $(n=264)$ than previous studies. We randomized participants to alcohol (0.6 and $0.51 \mathrm{~g} / \mathrm{kg}$ for males and females, resp.) or placebo, and assessed moral judgment using standard sacrificial dilemmas (trolley problems) thought to probe the interaction between emotional intuitions and controlled cognitive processes in moral cognition [25-28]. Prosocial behavior was assessed using modified versions of the dictator game $[29,30]$. For risk taking, we used two different tasks, covering both intuitive-cognitive aspects of decision making, via standard prospect theory gambles [31], and more affect-laden decisions from experience, using the Balloon Analog Risk Task (BART; [32]). Finally, waiting impulsivity was assessed using a prototypical task that captures participants' preferences for real monetary rewards delivered at different points in time [33, 34]. We assessed both general discounting (over relatively short delays) and temporal inconsistency in discounting, known as present bias, which is a characteristic property of discounting models that feature a sharp rise in the discounting rate for rewards delivered closer to today, such as quasi-hyperbolic discounting $[34,35]$.

\section{MATERIALS AND METHODS}

\section{Ethics statement}

The study was approved by the Regional Ethical Review Board of Linköping (ref 2016/496-31) and all participants provided written informed consent.

\section{Open science}

The preregistration together with data, analysis codes (main analyses), and experimental materials are available via the project's OSF repository (https://osf.io/sf5em). Individual level data for the main analyses are shown in Supplementary materials Fig. S1-S4. We preregistered six main questions of interest for this data collection; this paper is focused on the first four of them.

\section{Participants}

Healthy volunteers were recruited using advertisements in social media, flyers, and the Online Recruitment System for Economic Experiments

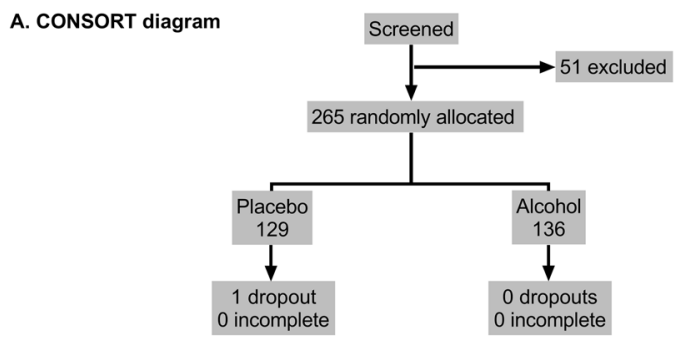

B. Study timeline
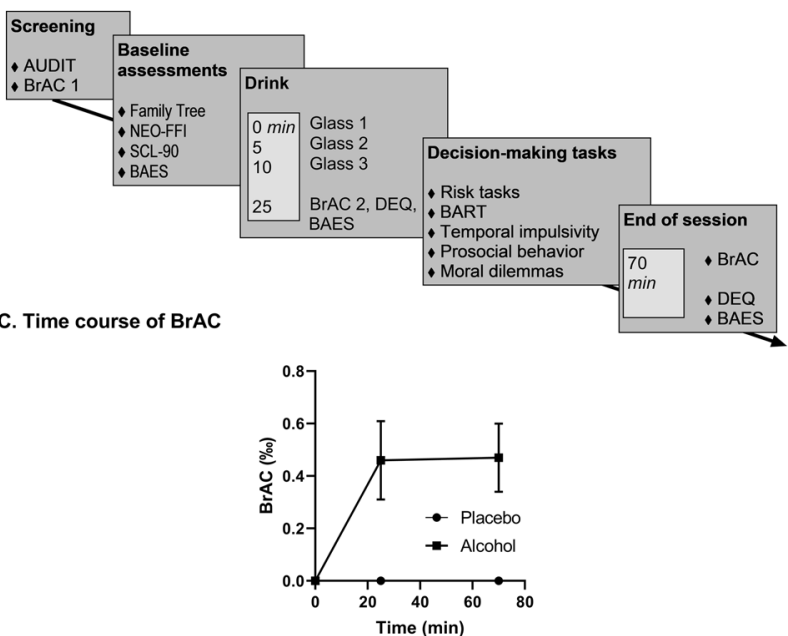

Fig. 1 Study outline. A CONSORT diagram of study participant. B study timeline. C time course of $\operatorname{BrAC}$ (mean \pm SD).

ORSEE [36] at Linköping University, Sweden. Eligible participants were randomized to alcohol $(n=128)$ or placebo $(n=136)$. The groups were similar in terms of baseline characteristics, including age, sex, education, alcohol consumption as measured with AUDIT, and personality traits measured with NEO-FFI (Table 1). The distribution of AUDIT scores was also very similar in both groups, and shown in Supplementary Materials (Fig. S1). Our final sample size is smaller than the pre-specified target of $\mathrm{n}=300$ because we had to stop enrolling participants due to the onset of the COVID-19 pandemic.

\section{Study timeline}

The study visit consisted of five phases (Fig. 1B): screening, questionnaires for baseline assessments, treatment phase (intake of drink), decisionmaking tasks performed at a computer, and a finishing phase with end of session questionnaires. The study was conducted in a computer lab in sessions of up to 15 participants, who were seated in separate cubicles and did not interact with each other.

\section{Screening and eligibility}

During the screening phase, prospective participants were evaluated for eligibility by a research nurse or a physician. Detailed eligibility criteria are provided in Supplementary Materials. In brief, subjects were excluded if they had any psychiatric disorder, were pregnant, had any previous neurological condition or if they were at risk of alcohol or other substance use disorders except nicotine. Alcohol Use Disorder Identification Test [AUDIT; [37]] was used to assess the presence of AUD or hazardous drinking. Weight and sex were noted. Breath alcohol concentration $(\mathrm{BrAC})$ baseline was measured using a breathalizer. A total of 316 individuals were evaluated, and 265 were included. Of these, 129 were allocated to placebo and 136 were assigned to alcohol (Fig. 1A).

\section{Baseline assessments}

Baseline personality traits were obtained using the NEO Five Factor Inventory [NEO-FFl; [38]]. The Symptom checklist-90 [SCL-90; [39]] was used to measure symptoms of anxiety and depression. The Family 
Tree Questionnaire [FTQ; [40]] was used to assess family history of alcohol problems. The Biphasic Alcohol Effect Scale [BAES; [41]] was used to measure stimulant and sedative effects of alcohol.

\begin{abstract}
Alcohol administration
Participants were informed that they would receive alcohol, corresponding to a $\mathrm{BrAC}$ of $0.6 \%$ or placebo, and were randomized to one of these in a parallel group design (see Fig. $1 \mathrm{~A}$ ). In the alcohol group, male participants received a $0.6 \mathrm{~g} / \mathrm{kg}$ dose of alcohol using a $12 \%$ solution. The solution was made using $95 \%$ ethanol mixed with cranberry juice. To adjust for known differences in body water, women received $85 \%$ of the alcohol administered to men. In the placebo group participants received a $1 \%$ alcohol solution. In both groups, the drink was divided into three glasses. Participants in both the alcohol and placebo group were required to finish each glass within five minutes. After the last glass, participants had a break for $15 \mathrm{~min}$ before proceeding with the decision-making tasks. Breath alcohol concentration (BrAC) was measured at baseline, 25 min later, just before the decision-making tasks and after additional appr. $45 \mathrm{~min}$, as soon as the participant finished the session. The Biphasic Alcohol Effect Scale [BAES; [41]] was performed every time BrAC was measured and the Drug Effect Questionnaire [DEQ; [42]] was measured the second and third time BrAC was measured.
\end{abstract}

\section{Decision-making tasks}

For detailed task description and instructions, see Supplementary Materials. In brief, tasks focused on four domains of decision making: waiting impulsivity, choice under risk, moral judgment, and prosocial behavior. Tasks were presented on a computer screen using Qualtrics and Inquisit software. Divider screens prevented participants from seeing each other's responses. Tasks were presented in a block-randomized order. At the end of the experiment, one decision for each subject was randomly selected and paid out for real (using the cell phone payment system Swish) together with the show-up fee of 150 SEK (appr. \$15) that participants received for participating in the study.

Waiting impulsivity. This was assessed using a prototypical task that measures participants' preferences for rewards delivered at different points in time $[33,34]$. Participants chose repeatedly between smaller rewards delivered sooner (SS) and larger rewards delivered later (LL). We tested for two distinct types of discounting; a general form of impatience, based on the proportion of smaller-sooner choices each person made in the first block of items (pr. smaller-sooner), and a specific form of impatience known as present bias, which is based on the difference (for each participant) between choices made in the first and second blocks of items (diff. pr. smaller-sooner). Present bias is a characteristic property of discounting models that feature a sharp rise in the discounting rate for rewards delivered closer to today, such as quasihyperbolic discounting $[34,35]$.

Risk taking. One of the tasks to examine risk taking used standard prospect-theory gambles [31]. We used incentivized binary choices between a lottery and a certain amount of money in three different domains: gain, loss, mixed. We used the proportion of choices where the gamble was our main dependent variable for each domain ( $p$ r. risky choices). Using this task enabled us to characterize choices after the expected patterns of prospect theory [31], which emphasizes greater risk aversion for gains than losses and disproportionate weighting of the loss component in mixed prospects.

The second task in this domain was the Balloon Analog Risk Task [BART; [32]], in which participants were presented with a picture of a balloon and could earn money by pumping up the balloon by clicking a button. Each click earned them 0.1 SEK and caused the balloon to incrementally inflate. If the balloon was overinflated, it exploded, and all money earned for that trial was lost. If instead participants had chosen to cash-out prior to the balloon exploding, the money earned for that trial was added to their sum for this task. Our main dependent variable was the average number of pumps per trial, excluding trials where the balloon exploded (avg. pumps per balloon).

Moral judgment. This was assessed using four sacrificial moral dilemmas (trolley problems) that involved a conflict between utilitarian and deontological moral foundations $[25,43,44]$. In each dilemma, participants were faced with the possibility of saving a certain number of people by sacrificing one individual. Killing the single person while saving the others is consistent with utilitarian judgment, while not pulling the switch is consistent with deontological judgment, whereby actively causing harm to another person is morally unacceptable regardless of overall consequences. The main dependent variable for moral judgment was based on participants' responses to four moral dilemmas (switch, footbridge, fumes, and shark; see Supplementary materials for details), presented in random order, and calculated as the proportion of utilitarian choices made by each participant (pr. utilitarian choices).

Prosocial behavior. This was assessed using two different tasks, designed to measure both altruistic behavior and preference for equality versus efficiency in distributions. Both were modified versions of the dictator game $[29,30]$.

In the first task, participants were endowed with 50 SEK (appr. \$5) and decided how much of it to keep for themselves and how much to donate to a well-known charity organization (Swedish Heart-Lung Foundation). The main dependent variable was the amount donated (donation to charity).

In the second task, subjects chose repeatedly between binary allocations of money (for themselves and another anonymous participant). Each item featured a choice between an equal distribution and an unequal but more efficient distribution, for example 40 SEK (appr. \$4) each vs 40 SEK for me and 50 SEK for the other participant. We used the proportion (for each person) of choices where the equal allocation was chosen over the more efficient allocation ( $p r$. equality).

\section{Statistical analysis}

The main analysis plan was specified before data collection begun, see the preregistration for details. STATISTICA 13.0 (Dell Inc, Tulsa, OK) was used for all analyses. One-way ANOVA, with group (alcohol or placebo) as a between-subject factor, and a pre-set alpha $=0.05$, were the preregistered main tests. Subject-level data for main tests are provided in Supplementary Materials, Fig. S1-S4. Secondary analyses (not preregistered) additionally assessed the potential influence of baseline subject characteristics (age, sex, personality measures, and alcohol use as measured by the AUDIT). Covariates were retained in analysis models if they were a significant predictor, or if they reduced the residual variance by more than $10 \%$; otherwise, they were excluded. In additional analyses (also not preregistered) we compared self-reported effects of alcohol (stimulant, sedative, strength of drug effect, desirability) across the two conditions, based on subjects' responses to the Biphasic Alcohol Effect Scale (BAES) and the Drug Effect Questionnaire (DEQ).

\section{RESULTS}

No BrAC alcohol was detected in the placebo group at any timepoint, or in the alcohol group at baseline. In the alcohol group, a BrAC of appr. $0.5 \%$ o was reached by the time behavioral testing started, and remained stable at that level until completion of testing (Fig. 1C). Using the Biphasic Alcohol Effects Scale [41], the alcohol group showed the expected stimulant as well as sedative effects of alcohol compared to the placebo group. On the Drug Effects Questionnaire [42], there was a clear effect of alcohol on the "Feel drug" and "High" items (Fig. 2). Neither "Like" nor "Want more" items were affected. The proportion of participants who correctly guessed their allocation was $95.5 \%$ in the alcohol group, and $69 \%$ in the placebo group. No unexpected adverse events were noted.

\section{Moral judgment in sacrificial dilemmas}

Preference for utilitarian responding was increased in the alcohol group (one way ANOVA: $\mathrm{F}_{1,262}=5.71, p=0.02$; Cohen's $\mathrm{d}=0.29$; Fig. $3 A$ ). This remained unchanged when controlling for potential confounds. In the final ANCOVA, agreeableness $(p<0.01)$, gender $(p=0.06)$ and hazardous alcohol use, as measured with the AUDIT ([37]; $p=0.02$ ) contributed to the model, and all correlated negatively with utilitarian choices. Exploratory analyses indicated that the effect of alcohol on moral judgment was driven by the switch and fumes dilemmas, and to some extent the shark dilemma, while no corresponding effect was seen in the footbridge dilemma. 
A. Feeling effect of drug

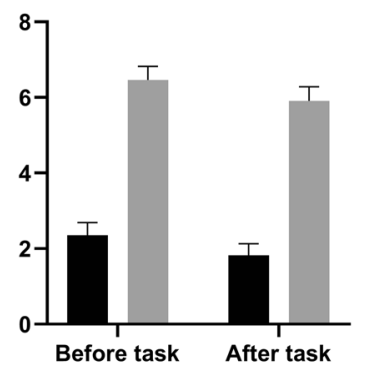

C.

Like

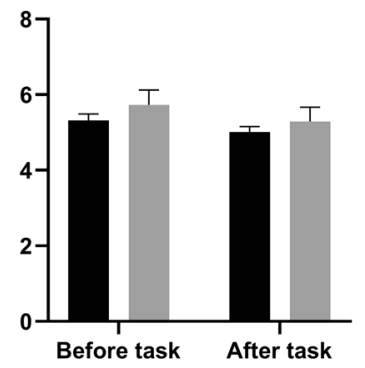

E.

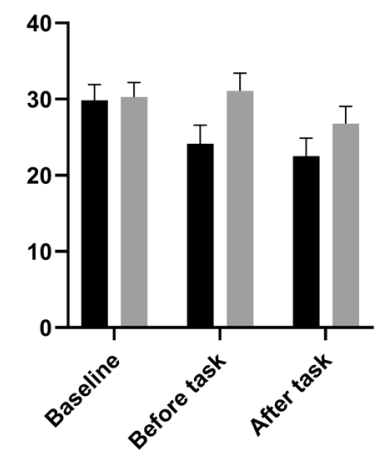

B.

$$
\text { Feeling high }
$$

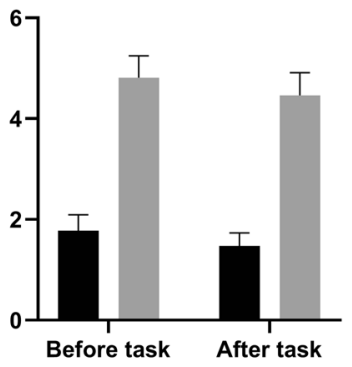

D. Want more

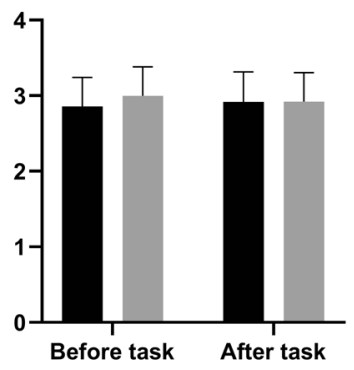

F.

Sedative

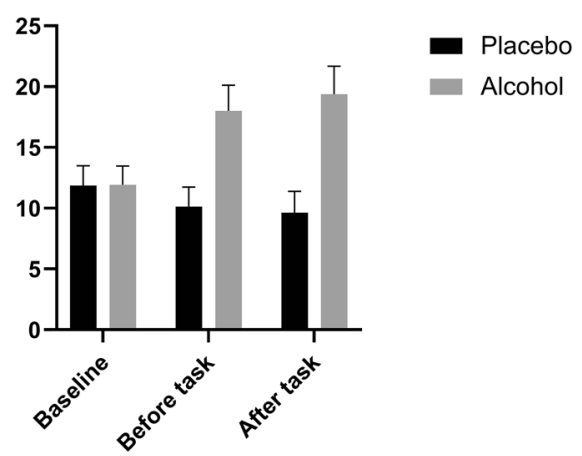

Fig. 2 Self-reported effects of alcohol. A-D Mean responses on the Drug Effect Questionnaire (DEQ) before and after the decision-making tasks. Error bars indicate $95 \%$ Confidence Intervals. E, F Mean responses to the Biphasic Alcohol Effects Scale (BAES). Error bars indicate $95 \%$ Confidence Intervals. Significant alcohol effects for all items are indicated in the Results section.

\section{Prosocial behavior}

Participants in the alcohol group donated more money to a charity $\left(F_{1,262}=4.83, p=0.03\right.$; Cohen's $d=0.27$; Fig. $\left.3 \mathrm{~B}\right)$. This remained unchanged when controlling for potential confound of baseline subject characteristics. In the final model, agreeableness $(p<0.01)$ and hazardous alcohol use as measured with the AUDIT $(p=0.02)$ significantly contributed to the model. Agreeableness was positively correlated with donations and AUDIT was negatively correlated.

Equality/efficiency tradeoffs did not differ between groups $\left(0.27 \pm 0.38\right.$ vs. $\left.0.27 \pm 0.39 ; F_{1}, 262<0.01, p=0.98\right)$; thus, participants in both groups were reluctant to pursue equality of resources if redistribution had a cost. This result remained unchanged when controlling for potential confounds. In the final model, age $(p<0.01)$, neuroticism $(p<0.01)$, extraversion $(p<0.01)$, openness $(p=0.02)$, conscientiousness $(p=0.01)$ and gender $(p<0.01)$ significantly contributed to the model. Openness correlated negatively with equality. Female gender, age, neuroticism, extraversion and conscientiousness correlated positively with equality.

\section{Risk taking - prospect theory gambles \& BART}

Behavior in the prospect gambles was similar in the two groups (Fig. 4). There was a tendency for decreased risk taking in the alcohol group for gains $\left(0.59 \pm 0.29\right.$ vs. $0.65 \pm 0.22 ; F_{1,262}=3.58$, $p=0.06)$, but no effect, or trend in the loss $(0.49 \pm 0.22$ vs. $0.45 \pm$ $\left.0.22 ; F_{1}, 262=1.72, p=0.19\right)$, or in the mixed domain $(0.49 \pm 0.21$ vs. $0.47 \pm 0.22 ; F_{1}, 262=0.64, p=0.42$ ). When all three domains were combined, the alcohol and placebo groups were virtually indistinguishable $\left(0.52 \pm 0.18\right.$ vs. $0.52 \pm 0.15 ; F_{1,262}<0.01, p=0.96$; Cohen's $\mathrm{d}=-0.01)$. This remained unchanged when controlling for potential confounds. In the final model, age $(p<0.01)$, 


\section{A. Moral judgment}
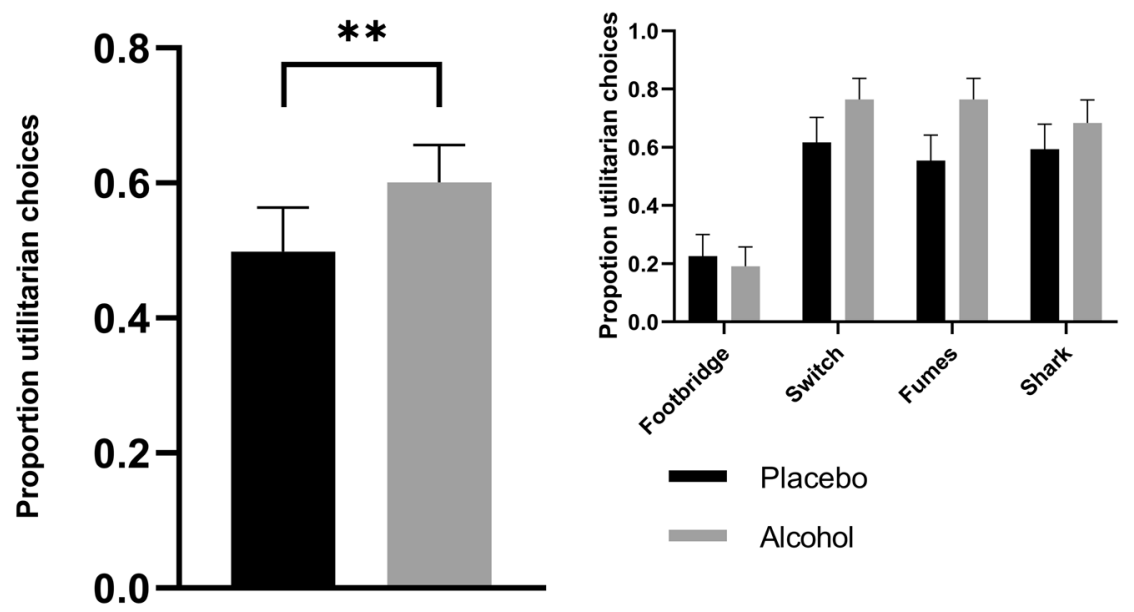

\section{B. Donation to charity}
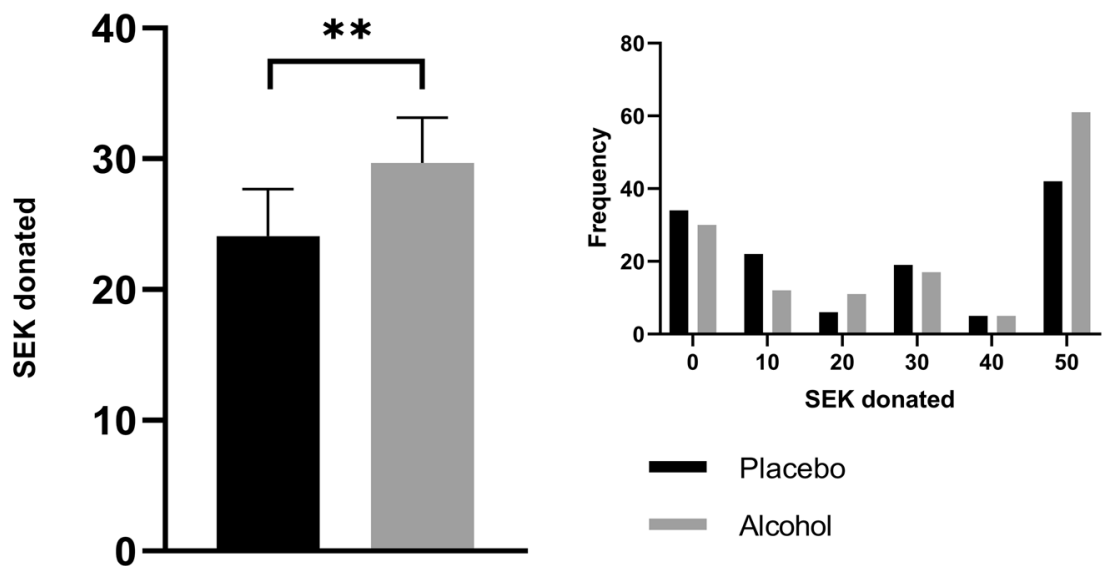

Fig. 3 Alcohol promotes utilitarian moral decisions and altruistic behavior. A Moral judgment. Main panel: overall proportion of utilitarian choices. Inset: proportion of participants in each group who chose the utilitarian option, for the respective scenario. B Donation to charity. Main panel: Total amount of money donated. Inset: distribution of amounts donated to the charity, by group. Ten Swedish kronor (SEK) was approximately equal to one USD at the time of the experiment. Tick marks on the $x$-axis show the midpoints of equally-sized bins (10 SEK wide), except at the endpoints, where bin size is smaller. Error bars indicate $95 \%$ Confidence Intervals. Sample size is $n=128$ for placebo and $n=136$ for alcohol.

extraversion $(p=0.01)$, conscientiousness $(p=0.03)$ and agreeableness $(p=0.06)$ significantly contributed to the model or showed a tendency to do so. Age and extraversion were positively correlated with risk taking, while agreeableness and conscientiousness were negatively correlated with risk taking.

Similarly, there was no difference in risk taking on the Balloon Analog Risk Task (BART) between alcohol and placebo (Fig. 4; $43.4 \pm$ 14.1 vs. $43.5 \pm 14.2 ; F_{1.257}<0.01, p=0.99$; Cohen's $\left.d=-0.002\right)$. This remained unchanged when controlling for potential confounds. In the final model, neuroticism $(p=0.01)$ and conscientiousness ( $p=$ 0.05 ) were significant covariates. Both were negatively correlated with adjusted average number of pumps.

\section{Waiting impulsivity}

There was no statistically significant difference between groups for waiting impulsivity $\left(0.24 \pm 0.31\right.$ vs. $0.29 \pm 0.31 ; F_{1,262}=2.21$, $p=0.14)$, or present bias $\left(0.0007 \pm 0.15\right.$ vs. $0.03 \pm 0.18 ; F_{1,262}=$ $2.59, p=0.11)$. Results were similar when all individual decisions were combined $\left(0.24 \pm 0.30\right.$ vs. $0.28 \pm 0.29 ; F_{1,262}=1.25, p=0.26$;
Cohen's $d=-0.14)$. Thus, any possible effect of alcohol on waiting impulsivity was small and insignificant, and the bound on the $95 \%$ confidence interval in the hypothesized direction, i.e., increased waiting impulsivity following alcohol intake, was close to zero. These results remained unchanged when controlling for potential confounds.

\section{DISCUSSION}

We conducted a large, preregistered RCT to assess acute effects of alcohol on measures of decision making in personal and social domains. A $0.6 \mathrm{~g} / \mathrm{kg}$ dose of alcohol did not influence personal decisions, but robustly moderated social decision making. In particular, subjects in the alcohol group showed an increased utilitarian preference in sacrificial moral dilemmas, and donated more money to charity in a modified dictator-game task. As an internal validation of these findings, we detected the expected effects of personality traits, independently of the alcohol effects. Although participants' level of alcohol use, as measured by the 
A.

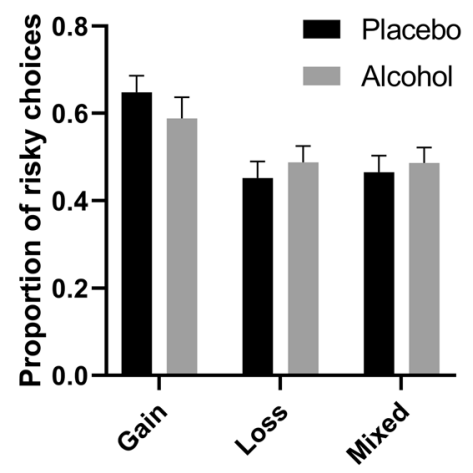

B.

\section{BART}

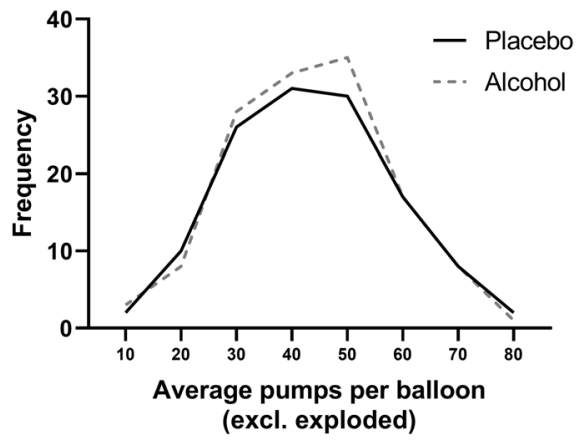

Fig. 4 Alcohol had no effect on risk taking. A Mean proportion of trials where individuals chose the gamble over the certain option, separated by domain (gain, loss, mixed). Error bars indicate $95 \%$ Confidence Intervals calculated from $t$ tests. B Distribution of the average number of pumps per balloon on the Balloon Analog Risk Task (BART). Sample size is $n=128$ for placebo and $n=136$ for alcohol, except for BART where two individuals in placebo and three in alcohol could not participate in the task due to software issues.

AUDIT scale, correlated negatively both with their utilitarian decisions and charitable donations, the effects of alcohol on these outcomes did not interact with the level of alcohol use, and thus did not differ across the spectrum of use included in the study. For personal decision making, we did not find an effect of alcohol at the dose given on any of several risk-taking measures or waiting impulsivity. As an internal validation, we reliably replicated known patterns of results with all our tasks, e.g., increased risk seeking for losses and selective sensitivity to harmful actions across different moral dilemmas. Thus, our null findings are unlikely a result of compromised task calibration or unusual sample composition. Our findings are also unlikely to be explained by effects on elements of decision making that are related to impulse control, since, at the moderate level of alcohol intoxication used, we found no effects in tasks specifically designed to capture this dimension of behavior.

Our results for moral judgment, that subjects became increasingly utilitarian, differ from the few previous studies. Francis and colleagues [21] recently conducted a placebo-controlled study on moral judgment, using both traditional moral dilemmas and an adapted virtual-reality moral behavior task. They found no effects of alcohol on any of these tasks. In contrast, Duke and Bègue [22] found that alcohol intake correlated with increased utilitarian responding, but only on the footbridge dilemma and not on the switch dilemma, in a study conducted at two bars in France. However, the results from these two studies should be interpreted with caution, given the small sample sizes and the correlational nature of the data in the latter study. Our findings are contrary to what would be expected based on the widely held dual-process theory of moral cognition $[25,28]$. According to this theory, the effects of alcohol to increase emotional reactivity and weaken cognitive control should give increased preference for deontological rather than utilitarian actions. In fact, we find the opposite, i.e. increased utilitarian responding due to alcohol. A possible account of this finding is that acute alcohol intoxication primarily affects moral judgment through effects on its cognitive elements, and does so by subtly shifting the balance between perceived costs and benefits in the utilitarian calculation. This is broadly consistent with findings indicating an important role of frontocortical brain areas in social decision making [45], and a higher sensitivity of these neocortical structures to alcohol effects compared to subcortical brain structures that generate incentive salience and affective signals [1].

Acute effects of alcohol on altruistic behavior using real monetary rewards have hardly been assessed at all previously. Two previous studies found no effect or a tendency for a negative effect on altruism following alcohol intake $[19,20]$. In contrast, we found that alcohol made people more altruistic, donating a larger proportion of their money (around ten percentage points more than the placebo group) to charity. This is a modest effect size, but appears to be highly specific, as it was found at a modest dose of alcohol at which there were no discernible effects on impulsivity or risk taking. We had no a priori expectation about the direction of the effect on altruism. In principle, these results can also be rationalized using alcohol myopia theory [46-48], which emphasizes impaired attention and thus increased reliance on salient stimuli following acute alcohol intoxication. The need of the charity recipients is arguably a salient cue in the task that we used, and it is possible that this is what caused increased donations in the alcohol group.

Previous studies on personal decision making for risk and impulsivity have found mixed results $[10-20,49]$, but most studies have been limited by a small sample size. Prior to our study, Bernhardt et al. [10] was probably the most well-powered study to date ( $n=54$ adolescent males in a within-subject design), and their results are similar to what we found, with no effects on waiting impulsivity or on risk taking in gain, loss, or mixed domains. Taken together, this strongly suggests that alcohol taken at moderated doses by healthy social drinkers has small or no effects on risk taking or waiting impulsivity. For the Balloon Analog Risk Task (BART), we are aware of only one previous study that was adequately powered, Rose et al. [50] with $n=142$ in a betweensubjects design; e.g., all other studies reviewed by Harmon et al. [51] had $<33$ subjects per treatment cell. Interestingly, whereas Rose et al. found increased risk taking (more pumps) due to alcohol intake (Cohen's $\mathrm{d}=0.40$ at a $0.6 \mathrm{~g} / \mathrm{kg}$ dose of alcohol), our results clearly favored a no-effects interpretation, with the $95 \%$ confidence interval bounded at an effect size or appr. Cohen's $d=$ 0.25 in either direction. Thus, more studies are needed to determine the acute effects alcohol on the BART. Of note, while the BART is commonly viewed as a generic "risk taking task", its original evaluation suggested that it may in fact be more related to sensation seeking and impaired behavioral inhibition [32], i.e. facets of the impulsivity distinct from those involved in trading off the magnitude of gains or losses vs. their probability.

Our study has several strengths as well as limitations. Among the former, it had a large sample size and a preregistered analysis plan. This is important given that prior studies are for the most part small and without transparent control of analytical flexibility. The combination of small sample sizes, high analytical flexibility and publication bias has been a perfect storm for generating 
irreproducible findings [52-55]. However, despite a larger sample than previous studies, we had insufficient power to conduct otherwise relevant subgroup analyses, for example based on gender or quantitative traits, beyond using them as covariates in the analysis. For the same reason, we did not attempt to capture biphasic effects of alcohol. Finally, we were not able to control for expectation effects by adding more conditions, while blinding was not successful. These limitations may affect the generalizability of our findings.

Some features of the study are both strengths and limitations. For instance, we ensured a high degree of experimental control, at the expense of assessing the effects of alcohol in a standardized, sterile laboratory environment. As expected under these conditions, while self-ratings of intoxication ("feeling effect" and "high") were robustly influenced by alcohol, neither "liking" nor "wanting" ratings were affected. On one hand, this suggests that our findings are unlikely to be primarily driven by expectations, since expectations of alcohol effects are linked to experiencing alcohol in a naturalistic context. At the same time, alcohol effects on decision making under laboratory conditions may differ from those "in the wild". Similarly, although we make a distinction between personal and social decision making in terms of outcomes, all decisions in our study were taken in private in front of a computer. Thus, future studies could extend our findings by investigating the effects of alcohol on social decisions made in a public setting (e.g., observed by an audience), where social signaling and reputational concerns also come into play.

Designing the experiment, we emphasized task comprehension, and all decisions that involved money were incentivized (participants were paid for one randomly drawn decision at the end). Payments were implemented via a standard cell phone transfer system in order to circumvent concerns about differential transactions costs in the waiting-impulsivity task [56]. However, as a potential side effect, this made the larger-later option in this task more attractive than we had anticipated, resulting in a more than usual amount of upper censoring (people who chose the largerlater option for all trials) for this task. Our results for waiting impulsivity should be interpreted with this limitation in mind. Similarly, our finding that alcohol did not influence impulsivity, may not generalize to higher doses, or other populations. Also, even at the dose used, effects on impulsivity might be present in people with substance use disorders, externalizing psychopathology, or both.

The pattern of our results suggests that alcohol selectively moderates decision making in the social domain, at least for lowmoderate doses of alcohol. This is consistent with existing theory that emphasizes the dual roles of shortsighted information processing and salient social cues in shaping decisions under the influence of alcohol [46]. Our findings are obtained in social drinkers without any AUD, but have potentially important implications for attempts to understand the emergence of AUD. Most prior alcohol challenge studies have focused exclusively on personal decision making, but changes in social cognition, ultimately resulting in social marginalization and exclusion, are at the core of the addictive process $[57,58]$. It has recently been shown that communicating deontologically rather than utilitarian-motivated decisions may be more advantageous to signal trustworthiness as group member $[59,60]$. Impairments in the ability to signal trustworthiness caused by alcohol use could contribute to social marginalization. These alcohol-induced effects on social cognition are likely to interact with pre-existing vulnerabilities to influence social functioning. Our findings highlight the importance of taking the social dimension of decision making into account to better understand the process of developing AUD.

Taking a broader perspective, to policymakers and everyday decision-makers alike, it is useful to know that the influence of alcohol on decision making is sensitive to social cues. Whether alcohol is ultimately good or bad for people's decisions will likely depend on context. Perhaps surprisingly, from the narrow perspective of our sample and the specific tasks that we used, social outcomes were more advantageous among people who were given alcohol compared to people who were not.

\section{REFERENCES}

1. Xiao P, Dai Z, Zhong J, Zhu Y, Shi H, Pan P. Regional gray matter deficits in alcohol dependence: a meta-analysis of voxel-based morphometry studies. Drug Alcohol Depend. 2015;153:22-28.

2. Boileau I, Assaad JM, Pihl RO, Benkelfat C, Leyton M, Diksic M, et al. Alcohol promotes dopamine release in the human nucleus accumbens. Synapse. 2003; 49:226-31.

3. Gilman JM, Ramchandani VA, Davis MB, Bjork JM, Hommer DW. Why we like to drink: a functional magnetic resonance imaging study of the rewarding and anxiolytic effects of alcohol. J Neurosci 2008;28:4583-91.

4. Urban NB, Kegeles LS, Slifstein M, Xu X, Martinez D, Sakr E, et al. Sex differences in striatal dopamine release in young adults after oral alcohol challenge: a positron emission tomography imaging study with $[(11) \mathrm{C}]$ raclopride. Biol Psychiatry. 2010;68:689-96.

5. Ramchandani VA, Umhau J, Pavon FJ, Ruiz-Velasco V, Margas W, Sun H, et al. A genetic determinant of the striatal dopamine response to alcohol in men. Mol Psychiatry. 2011;16:809-17.

6. Fairbairn CE, Sayette MA. The effect of alcohol on emotional inertia: a test of alcohol myopia. J Abnorm Psychol 2013;122:770-81.

7. Sayette MA, Creswell KG, Dimoff JD, Fairbairn CE, Cohn JF, Heckman BW, et al. Alcohol and Group Formation: A Multimodal Investigation of the Effects of Alcohol on Emotion and Social Bonding. Psychol Sci 2012;23:869-78.

8. CE Fairbairn, D Kang, KD Federmeier, Alcohol and neural dynamics: a metaanalysis of acute alcohol effects on event-related brain potentials. Biol Psychiatry https://doi.org/10.1016/j.biopsych.2020.11.024 (2020).

9. Herman AM, Duka T. Facets of impulsivity and alcohol use: what role do emotions play? Neurosci Biobehav Rev 2019;106:202-16.

10. Bernhardt N, Obst E, Nebe S, Pooseh S, Wurst FM, Weinmann W, et al. Acute alcohol effects on impulsive choice in adolescents. J Psychopharmacol. 2019;33: 316-25.

11. Bidwell LC, MacKillop J, Murphy JG, Grenga A, Swift RM, McGeary JE. Biphasic effects of alcohol on delay and probability discounting. Exp Clin Psychopharmacol. 2013;21:214-21.

12. Balodis IM, MacDonald TK, Olmstead MC. Instructional cues modify performance on the lowa Gambling Task. Brain Cogn. 2006;60:109-17.

13. Lane SD, Cherek DR, Pietras CJ, Tcheremissine OV. Alcohol effects on human risk taking. Psychopharmacol. 2004;172:68-77.

14. Richards JB, Zhang L, Mitchell SH, de Wit H. Delay or probability discounting in a model of impulsive behavior: effect of alcohol. J Exp Anal Behav. 1999;71:121-43.

15. Reynolds B, Richards JB, de Wit H. Acute-alcohol effects on the Experiential Discounting Task (EDT) and a question-based measure of delay discounting. Pharmacol Biochem Behav 2006;83:194-202.

16. Ortner CN, MacDonald TK, Olmstead MC. Alcohol intoxication reduces impulsivity in the delay-discounting paradigm. Alcohol Alcohol. 2003;38:151-6.

17. Adams S, Attwood AS, Munafò MR. Drinking status but not acute alcohol consumption influences delay discounting. Hum Psychopharmacol. 2017;32:e2617.

18. George S, Rogers RD, Duka T. The acute effect of alcohol on decision making in social drinkers. Psychopharmacol. 2005;182:160-9.

19. Corazzini L, Filippin A, Vanin P. Economic behavior under the influence of alcohol: an experiment on time preferences, risk-taking, and altruism. PLoS ONE. 2015;10: e0121530.

20. Bregu K, Deck C, Ham L, Jahedi S. The effects of alcohol use on economic decision making. South Economic J. 2017;83:886-902.

21. Francis KB, Gummerum M, Ganis G, Howard IS, Terbeck S. Alcohol, empathy, and morality: acute effects of alcohol consumption on affective empathy and moral decision-making. Psychopharmacol. 2019;236:3477-96.

22. Duke $A A$, Bègue $L$. The drunk utilitarian: blood alcohol concentration predicts utilitarian responses in moral dilemmas. Cognition. 2015;134:121-7.

23. Burghart DR, Glimcher PW, Lazzaro SC. An expected utility maximizer walks into a bar.... J risk Uncertain. 2013;46:215-46.

24. Proestakis A, Espín AM, Exadaktylos F, Cortés Aguilar A, Oyediran OA, Palacio LA. The separate effects of self-estimated and actual alcohol intoxication on risk taking: a field experiment. J Neurosci Psychol Econ. 2013;6:115-35.

25. Greene JD, Nystrom LE, Engell AD, Darley JM, Cohen JD. The neural bases of cognitive conflict and control in moral judgment. Neuron. 2004;44:389-400.

26. Tinghög $G$, Andersson D, Bonn $C$, Johannesson $M$, Kirchler $M$, Koppel $L$, et al. Intuition and moral decision-making - the effect of time pressure and cognitive load on moral judgment and altruistic behavior. PLoS ONE. 2016;11:e0164012. 
27. Persson $E$, Heilig M, Tinghög G, Capusan AJ. Using quantitative trait in adults with ADHD to test predictions of dual-process theory. Sci Rep. 2020;10:20076.

28. JD Greene, L Young, "The cognitive neuroscience of moral judgment and decision-making" in The Cognitive Neurosciences, D Poeppel, GR Mangun, MS Gazzaniga, Eds. (MIT Press, 2020).

29. Forsythe R, Horowitz JL, Savin NE, Sefton M. Fairness in simple bargaining experiments. Games Economic Behav. 1994;6:347-69.

30. Kahneman D, Knetsch JL, Thaler R. Fairness as a constraint on profit seeking: entitlements in the market. Am Economic Rev. 1986;76:728-41.

31. Kahneman D, Tversky A. Prospect theory: an analysis of decision under risk. Econometrica. 1979;47:263-91.

32. Lejuez CW, Read JP, Kahler CW, Richards JB, Ramsey SE, Stuart GL, et al. Evaluation of a behavioral measure of risk taking: the Balloon Analogue Risk Task (BART). J Exp Psychol Appl. 2002;8:75-84.

33. Hariri AR, Brown SM, Williamson DE, Flory JD, de Wit H, Manuck SB. Preference for immediate over delayed rewards is associated with magnitude of ventral striatal activity. J Neurosci. 2006:26:13213-7.

34. McClure SM, Laibson DI, Loewenstein G, Cohen JD. Separate neural systems value immediate and delayed monetary rewards. Science. 2004;306:503-7.

35. Laibson D. Golden eggs and hyperbolic discounting. Q J Econ. 1997;112:443-78

36. Greiner B. Subject pool recruitment procedures: organizing experiments with ORSEE. J Economic Sci Assoc. 2015;1:114-25.

37. Saunders JB, Aasland OG, Babor TF, de la Fuente JR, Grant M. Development of the Alcohol Use Disorders Identification Test (AUDIT): WHO Collaborative Project on Early Detection of Persons with Harmful Alcohol Consumption-Il. Addiction. 1993;88:791-804.

38. PT Costa, RR McCrae, Revised NEO personality inventory (NEO PI-R) and NEP fivefactor inventory (NEO-FFI): professional manual (Psychological Assessment Resources, Odessa, Fla. (P.O. Box 998, Odessa 33556), 1992), pp. vi, 101 p.

39. L Derogatis, SCL-90-R: Manual-II (Clinical Psychmetric Research, Towson, MD, 1983).

40. Mann RE, Sobell LC, Sobell MB, Pavan D. Reliability of a family tree questionnaire for assessing family history of alcohol problems. Drug Alcohol Depend. 1985; 15:61-67.

41. Martin CS, Earleywine M, Musty RE, Perrine MW, Swift RM. Development and validation of the Biphasic Alcohol Effects Scale. Alcohol Clin Exp Res. 1993;17:140-6.

42. Morean ME, de Wit H, King AC, Sofuoglu M, Rueger SY, O'Malley SS. The drug effects questionnaire: psychometric support across three drug types. Psychopharmacol. 2013;227:177-92.

43. Thomson JJ. The trolley problem. Yale Law J. 1985;94:1395-415.

44. P Foot, Virtues and Vices and Other Essays in Moral Philosophy (Clarendon, Oxford. First published in 1978 by Blackwell publisher and University of California press, 2002).

45. Knoch D, Pascual-Leone A, Meyer K, Treyer V, Fehr E. Diminishing reciprocal fairness by disrupting the right prefrontal cortex. Science. 2006;314:829-32.

46. Steele CM, Josephs RA. Alcohol myopia. Its prized and dangerous effects. Am Psychol. 1990;45:921-33.

47. Steele CM, Southwick L. Alcohol and social behavior I: The psychology of drunken excess. J Pers Soc Psychol. 1985;48:18-34.

48. Steele CM, Critchlow B, Liu TJ. Alcohol and social behavior II: the helpful drunkard. J Pers Soc Psychol. 1985;48:35-46.

49. Breslin FC, Sobell MB, Cappell H, Vakili S, Poulos CX. The effects of alcohol, gender, and sensation seeking on the gambling choices of social drinkers. Psychol Addict Behav. 1999;13:243-52.

50. Rose AK, Jones A, Clarke N, Christiansen P. Alcohol-induced risk taking on the BART mediates alcohol priming. Psychopharmacol. 2014;231:2273-80.

51. Harmon DA, Haas AL, Peterkin A. Experimental tasks of behavioral risk taking in alcohol administration studies: a systematic review. Addict Behav. 2021;113:106678

52. Open Science Collaboration, Estimating the reproducibility of psychological science. Science 349 (2015).
53. Munafò MR, Nosek BA, Bishop D, Button KS, Chambers CD, du Sert NP, et al. A manifesto for reproducible science. Nat Hum Behav. 2017;1:0021.

54. loannidis JPA. Why most published research findings are false. PLoS Med. 2005;2: e124.

55. Begley CG, Ellis LM. Raise standards for preclinical cancer research. Nature. 2012;483:531-3.

56. Cohen JD, Ericson KM, Laibson D, White JM. Measuring time preferences. J Econ Lit. 2020;58:299-347.

57. Heilig M, Epstein DH, Nader MA, Shaham Y. Time to connect: bringing social context into addiction neuroscience. Nat Rev Neurosci. 2016;17:592-9.

58. Heilig M, MacKillop J, Martinez D, Rehm J, Leggio L, Vanderschuren LJMJ. Addiction as a brain disease revised: why it still matters, and the need for consilience. Neuropsychopharmacology. 2021. https://doi.org/10.1038/s41386-020-00950-y.

59. Sacco DF, Brown M, Lustgraaf CJN, Hugenberg K. The adaptive utility of deontology: deontological moral decision-making fosters perceptions of trust and likeability. Evolut Psychological Sci. 2017;3:125-32.

60. Everett JA, Pizarro DA, Crockett MJ. Inference of trustworthiness from intuitive moral judgments. J Exp Psychol Gen. 2016;145:772-87.

\section{ACKNOWLEDGEMENTS}

We are grateful to Åsa Axén, Sandra Boda, Sarah Gustavson, Lisbet Severin, Lina Koppel, Theodor Arlestig and David Andersson for assisting with data collection.

\section{AUTHOR CONTRIBUTIONS}

$\mathrm{MH}$ and GT provided funding for the study. HK, EP, IP, MH, and GT designed the study. HK, AY and GT collected the data. HK and EP analyzed the data and drafted the manuscript. All authors revised the manuscript and approved the final manuscript for submission.

\section{FUNDING INFORMATION}

This work was supported by the Swedish Research Council (MH: 2013-07434; GT: 2018-01755) and the Swedish Research Council for Health, Working Life and Welfare (EP: 2020-00864). Funders had no role in study design, data collection, analysis, decision to publish, or preparation of the manuscript.

\section{COMPETING INTERESTS}

$\mathrm{MH}$ has received consulting fees, research support or other compensation from Indvior, Camurus, BrainsWay, Aelis Farma, and Janssen Pharmaceuticals. All other authors declare no conflicting interests.

\section{ADDITIONAL INFORMATION}

Supplementary information The online version contains supplementary material available at https://doi.org/10.1038/s41386-021-01218-9.

Correspondence and requests for materials should be addressed to Markus Heilig.

Reprints and permission information is available at http://www.nature.com/ reprints

Publisher's note Springer Nature remains neutral with regard to jurisdictional claims in published maps and institutional affiliations. 